\title{
ALGUNAS REFLEXIONES SOBRE EL PROCESO CONSTITUCIONAL EUROPEO*
}

\author{
Federico Fernández-Crehuet \\ Daniel García López \\ Universidad de Almería
}

RESUMEN. En este trabajo se examinan algunas cuestiones en torno al proceso de «constitucionalización» del Derecho comunitario. En primer término se abordan los problemas nominales que han acompañado al Tratado de Lisboa y a su precursora, la malograda "Constitución europea». En segundo lugar, se abunda en la denominada «tesis del no demos», que parece haber sido la vencedora, al menos a nivel del discurso teórico, tras la firma del Tratado de Lisboa. En la última parte del trabajo, se afronta de modo sucinto, la cuestión de la ciudadanía europea en relación con este proceso de constitucionalización. En general, la tesis central del trabajo puede ser resumida del siguiente modo: la Unión Europea no se puede subsumir ni explicar bajo los conceptos que se han empleado tradicionalmente para el Estado Nación.

Palabras clave: proceso constitucional europeo, ciudadanía europea, Estado Nación, Constitución europea, Tratado de Lisboa.

ABSTRACT. This paper examines some issues connected to the process of "constitutionalization» of European Community law. First, the denomination problems that have accompanied the Treaty of Lisbon and its predecessor, the ill-fated "European Constitution», are addressed. Second, the socalled «no demos thesis» is analyzed. This thesis seems to have been successful, at least at the level of theoretical discourse, following the signing of the Treaty of Lisbon. In the last part of the work, the question of European citizenship in relation to the constitutional process is addressed. In general, the central thesis of the work can be summarized as follows: the European Union cannot be subsumed or explained under the concepts used traditionally to refer to the Nation State.

Keywords: European constitutional process, European Citizenship, Nation State, European Constitution, Treaty of Lisbon.

* Fecha de recepción: 20 de mayo de 2008. Fecha de aceptación: 22 de julio de 2008. 


\section{¿SÓLO UNA CUESTIÓN NOMINAL?}

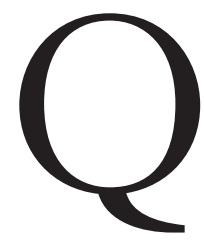

uizá exista en el tono agrio con que los internacionalistas responden cuando se emplea el término «Constitución europea», cierta dosis de verosimilitud: «No es una Constitución sino un Tratado», espetan. Uno de los argumentos principales que esgrimen es: «Los sujetos que lo han firmado son Estados». Las reformas en el texto, llevadas a cabo en Lisboa, parecen otorgarles la razón. Si se cuestiona el lugar hegemónico que ha de ocupar la Constitución nacional y se sitúa en la cúspide normativa a la «Constitución europea» también los constitucionalistas se muestran ásperos. Este tipo de reacciones - nos parece- pueden interpretarse como una obsesión por el discurso teórico (como si las palabras, por sí solas, fueran capaces de modificar la realidad o constituirla: algo típico de los juristas académicos), y, al mismo tiempo, una imagen, relativamente transparente de cómo debe estar diseñada la arquitectura de la Unión Europea. Es cierto que existen posturas más temperadas, que adoptan razonamientos eclécticos. P. HÄBERLE, hace ya algún tiempo, ha mostrado el proceso evolutivo por el que se rige la constitucionalización del Derecho europeo ${ }^{1}$. Algunos internacionalistas, por su parte, han señalado que el lenguaje, la función de la Constitución europea serían los propios de una «Constitución»; mientras que la forma coincidiría con la de un Tratado. No se trataría, en puridad, para algunos de estos autores, de una «Constitución verdadera» ${ }^{2}$.

En última instancia, la discusión (quizá excesivamente caricaturizada) está desenfocada. Ambas posturas parten de las estructuras y funciones de un «Estado-nación» «clásico» y «mito-tipificado», que ahora se habrían encarnado en otra realidad, la Unión Europea. Si nos es permitida la simplificación, los internacionalistas públicos tratan de analizar la Unión Europea con el mismo marco explicativo que cualquier otra relación exterior de un Estado-nación; los constitucionalistas intentan expandir los conceptos y estructuras del ordenamiento jurídico interno al exterior. En el seno de las filas de los constitucionalistas (de los no euro-escépticos), no es extraño encontrar malas interpretaciones de las tesis de J. FISCHER ${ }^{3}$. Según éstas, la Unión Europea sería idéntica a

1 P. HäBERLE, Europäische Rechtskultur, Frankfurt, Suhrkamp, 1997, p. 37.

2 M. JoOP y S. MATL, «Der Europäische verfassungvertrag als Höhepunkt im Prozess der Konstituionalisierung der EU», en M. Joop y S. MatL (eds.), Der Vertrag über eine Verfassung für Europa-Analysen zur Konstitutionalisierung der EU, Nomos Verlag, 2005, p. 38. MANGAS MARTín afirma que la Constitución europea no es una verdadera Constitución y escribe: «Ahora bien, con el término "Constitución europea" se ha buscado apelar a un valor político-cultural histórico propio de la tradición jurídica democrático-liberal europea y atraer a la ciudadanía hacia las normas fundamentales de un sistema jurídico y político decisivo para sus vidas». Hablar de «Constitución verdadera» es lo mismo que hablar de «derecho de propiedad verdadero», por ejemplo. Calificar a una institución jurídica como «verdadera o falsa» tras la llegada del historicismo al Derecho, es seguir creyendo en el «cielo de los conceptos jurídicos»; extremo que, dicho esto con todos los respetos, no se nos antoja muy afortunado. A. Mangas Martín, La Constitución europea, Iustel, 2005, sobre las «constituciones verdaderas», pp. 19 y ss. MACCORMICK, por su parte, habla de una Constitución encapsulada en un Tratado.

${ }^{3}$ FISCHER propone seguir la vía de SCHUMANN para crear una federación europea. Ahora bien, el discurso de la Humboldt presenta algunas matizaciones que se silencian. Señalaremos algunas. 1. Se subraya el predicamento de la tradición conservadora para Europa, convirtiendo el proyecto europeo en un espacio transpolítico. 2. Se resalta el valor que Francia y Alemania aquilatan en la construcción europea. 3. Se acentúa la necesidad de construir una Europa abierta hacia el Este. 4. El concepto de federación se matiza: «eine 
un Estado-nación de carácter federal; por ello, requeriría de una Constitución propia, que presentaría, a su vez, importantes concomitancias con las constituciones que han regulado el Estado-nación de carácter federal. En última instancia, aquellos que no son euro-escépticos parecen querer reducir el debate de la Constitución europea a una disyuntiva nominal (Tratado o Constitución) que incurre en el mismo error: se intenta explicar la Unión Europea por medio de los conceptos que durante cierto tiempo han servido para el Estado-nación.

Parece —insistimos, parece— que el Tratado de Lisboa les ha dado la razón a los internacionalistas y que estamos ante un Tratado internacional. ¿Pero no será que la sustitución del término Constitución es una estrategia? ¿Cambian los productos jurídicos por modificarle su nombre? MACCORMICK defendió la idea de que se trataba de una Constitución encapsulada en un Tratado.

Sinceramente creemos que estas dificultades serían menores, si se recordara que el término «Constitución» es polisémico y, sobre todo, histórico. Estos dos rasgos se retroalimentan. Desde el punto de vista de la estructura, de los contenidos y de las funciones existe un abismo jurídico entre las constituciones españolas de 1812 y la de 1978. Y, desde luego, las llamadas «constituciones», anteriores a la Revolución Francesa y a la Americana, carecían, según D. GRIMM, de ese carácter normativo, típico de las constituciones «modernas». El cambio de significado de los conceptos jurídicos no es un descubrimiento. Pero incluso la obviedad, a veces, se puede perder de vista.

Un modo de limar, al menos en parte, estas asperezas conceptuales sería esbozar una clasificación de los distintos tipos de «Constitución» que, históricamente, se han producido en el seno de los Estados-nación y observar las analogías y diferencias que presentan con el Tratado-Constitución europea.

Es mérito de G. FrANKENBERG haber diseñado una clasificación de las constituciones que, lejos de establecer taxones meramente sistemático-abstractos, tiene muy presente los acontecimientos históricos. Me parece, pues, que puede ser esclarecedora aplicada a la Constitución europea. Este autor diferencia entre 1) Constitución como contrato, 2) Constitución como manifiesto, 3) Constitución como programa, y 4) Constitución como ley. Creo que la Constitución europea posee elementos comunes a los dos primeros tipos.

schlanke und zugleich handlungsfähigs Europäische Föderation» (una federación europea delgada y, a la par, con capacidad de maniobra). 5. Se apuesta por la idea del Gravitationszentrum, tomando una considerable distancia del Kerneuropa (núcleo europeo), expresión acuñada por el conservador SCHÄUBLE. «Graviatationszentrum» es un término que hace alusión a la posibilidad de que el centro de Europa oscile, pivote, hacia unos u otros países europeos o incluso, como añade FISCHER, hacia países candidatos: «diese Antwangarde darf niemals exclusiv, sondern muss für alle mitgliedstaaten und Beitrittskandidaten der EU offen sein, wenn diese zu einem bestimmten Zeitpunkt teilnehmen wollen» (p. 252). Creo que, por estas y otras razones, no se puede aseverar que FISCHER defienda la construcción de una federación que deje al margen a los Estados-nación; él mismo lo afirma bien claro: «Dies alles wird aber nicht die Abschaffung des Nationalstaates bedeuten» (p. 249). J. FISCHER, «Vom Staatenverbund zur Föderation. Gedanken über die Finalität der europäischen Integration», en W. LOTH, Entwürfe einer Europäische Verfassung. Eine historische Bilanz, Europa Union Verlag, 2002, pp. 241 y ss. 


\section{CONSTITUCIÓN COMO CONTRATO}

El modelo Constitución-contrato puede ser confundido con el contrato social hobbesiano o roussoniano. Se puede establecer alguna diferencia. El contrato social versa sobre la creación hipotética de un contrato entre determinados individuos para establecer una voluntad común y, posteriormente, éste se ha de plasmar en un sistema político al que se le trasfiere el poder. Empero una «Constitución» no es un «contrato hipotético» para crear un Leviatán, ni un contrato social para establecer un autogobierno. Las constituciones son realidades históricas; se producen en un momento concreto y son una plasmación de ciertos intereses particulares. No son experimentos de teoría política.

Las constituciones-contrato se pueden caracterizar por:

a) El poder constituyente se recoge por medio de una «fórmula discreta», que parece diluirlo en la generalidad: «nosotros», «los firmantes», etc. Se trata usualmente de expresiones que plasman el contenido de la voluntad general, estructurando ésta, ya sea a través de los individuos o ya por medio de sujetos de carácter colectivo.

b) Es típico de las constituciones-contrato la distribución de competencias entre un gobierno institucionalizado y otros miembros que han participado, de modo directo o indirecto, en la instauración de la Constitución. Sobre todo, en ciertos momentos históricos, estas constituciones sirvieron para llevar a cabo el reparto de competencias entre monarcas y barones.

c) Usualmente se presupone que las partes del contrato-constitución son de carácter individual; normalmente ciudadanos que están unidos, por lazos jurídicos, a un territorio.

d) El acuerdo que se produce entre los ciudadanos o entre los nobles es eco de un elemento fundamental de la Constitución: la soberanía. Ésta implica, históricamente, una limitación de la summa potestas del Monarca; posteriormente se inscribe en el seno de la teoría de la separación de poderes.

e) Las constituciones-contrato suelen ser un modo de legitimación de la dominación política, en cuanto que ésta se constituye como auto-gobierno o auto-legislación y poseen, de otro lado, un carácter simbólico. Desde un punto de vista normativo, son poco exigentes: escaso articulado y organización defectuosa. Los criterios de integración son bastante débiles, y estos quedan reducidos a un criterio de organización política que se formula como legitimidad política o como procedimiento ${ }^{4}$.

El Tratado-Constitución europea podría ser, hasta cierto punto, revisada bajo la luz del taxón «constitución-contrato». Responde, ciertamente, a una filosofía del pacto y del «consenso», desde luego permeable al poder y al distinto peso de los diferentes Estados-nación. Es un «contrato real», pues de él se desprenden obligaciones jurídicas para las partes del «contrato». Sin embargo, como «constitución-contrato» presenta algunas peculiaridades:

a) Las «partes contratantes» son difusas. Aunque definidas concretamente en el momento del «contrato», las partes que lo han firmado pueden ser modificadas.

${ }^{4}$ G. FrankenberG, Autorität und Integration, Suhrkamp, 2003, pp. 79 y ss. 
No queda claro quién o quiénes pueden adherirse al «contrato inicial». No existe una definición clara de este extremo. Europa no parece poseer unas fronteras que sean inamovibles. Esto se deja notar en la construcción de la Unión Europea; mas no es un obstáculo definitivo para su construcción, es uno de sus rasgos constitutivos. El proceso de ensamblaje de la UE no ha tenido una meta clara, ha sido un proceso de «learning by doing». La incorporación de los distintos Estado-nación y de sus ciudadanos han dependido de factores políticos (la caída de las dictaduras de muchos de los países europeos), económicos (la construcción de un mercado que pudiera competir con otras grandes potencias), geoestratégicos (crear y mantener una situación de paz en Europa) y, por supuesto, sociológicos (la demanda de ciertas capas de la población, sobre todo, ciertas elites intelectuales y políticas de crear un espacio propio). La necesaria confluencia de todos estos factores puede haber ralentizado el proceso, pero no parece haberlo detenido.

b) En el Tratado-Constitución europea se aúnan las «partes del contrato» de carácter individual, los ciudadanos europeos — como sucedía en los Estados-nación en el momento de crear una Constitución - con los sujetos colectivos, los Estados-nación - como ocurre en el caso de los Tratados internacionales clásicos- ${ }^{5}$. Además los sujetos que constituyen ese «contrato-constitucional» poseen una relevancia heterogénea. El peso concedido a los Estado-nación es complejo y se modula dependiendo de las competencias. Esto hace que no se pueda subsumir este haz de relaciones bajo los contornos de una figura jurídica concreta, ya fuera ésta el Tratado internacional o la Constitución, sino que el Tratado constitucional adopta, a nuestro juicio, un carácter híbrido. Y ello, principalmente, causa que ya no sea posible utilizar el término clásico de soberanía; como argumentaremos parece más adecuado emplear la expresión «post-soberanía».

\section{CONSTITUCIÓN COMO MANIFIESTO}

Arquetipos de esta categoría serían la Declaración Francesa de los Derechos del Hombre y del Ciudadano de 1789, incorporada como Preámbulo a la Constitución francesa de 1791 y la Bill of Rights de Virginia. FRANKENBERG esquematiza la silueta de este tipo de textos normativos del siguiente modo:

a) Los manifiestos suelen ser declaraciones unilaterales que no tienen en cuenta la presencia del pueblo como poder constituyente. Estos plasman un tipo de «verdad histórica», que a veces parece ajena al pueblo.

b) La constituciones-manifiesto recogen tan sólo un conjunto limitado de valores, principios y fines (en algunos casos derechos), que se consideran fundamentales.

5 «La presente Constitución, que nace de la voluntad de los ciudadanos y de los Estados de Europa de construir un futuro común, crea la Unión Europea, a la que los Estados miembros atribuyen competencias para alcanzar sus objetivos comunes. 1. La Unión coordinará las políticas de los Estados miembros encaminadas a lograr dichos objetivos y ejercerá, de modo comunitario, las competencias que éstos le atribuyan. 2. La Unión está abierta a todos los Estados europeos que respeten sus valores y se comprometan a promoverlos en común». Art. 1 del Tratado por el que se establece una Constitución para Europa. Con el nuevo Tratado de Lisboa, que pasa del formato Tratado Constitucional al clásico Tratado reforma, estas referencias desaparecen. 
c) Las «constituciones-manifiesto» conceden cierta importancia a los aspectos estéticos-formales. Entre lo que dicen y lo que imponen existe un hiato. Una cosa son los derechos y fines que enuncian; otra la posibilidad de exigirlos por medios efectivos. A veces estos manifiestos se incorporan a otros textos que aquilatan mayor validez jurídica.

d) No pocas veces, debido al momento de su nacimiento, las «constitucionesmanifiesto» prestan una mayor atención a los derechos de los ciudadanos que a la organización política; sobre todo, cuando ésta hace referencia a procedimientos de decisión política ${ }^{6}$.

Efectivamente, el «Tratado-Constitución europea» posee algo de manifiesto, algo de acto simbólico, de acto constitutivo. Tal vez sea la «Carta de los Derechos fundamentales de la Unión Europea» junto con los valores que se incorporan en el preámbulo y en el art. I.2 lo que mejor plasma este carácter de «manifiesto», que no debe ser oponible al valor normativo de la Constitución. En el actual Tratado de Lisboa —que mantiene la esencia constitucional en la ambigüedad de la Declaración de Berlín y en las declaraciones de Angela Merkel ante el Parlamento Europeo ${ }^{7}$ como ejes que impulsaron la reforma- se preserva ese carácter de manifiesto desde su primer artículo, al incorporar al Preámbulo del Tratado de la Unión Europea el propio Preámbulo de la Constitución europea y al pasar el art. I.2 de la Constitución al art. 1 bis del TUE.

No se puede menguar, como a veces se suele hacer, el Tratado-Constitución europea: ésta no sólo es la Carta de los Derechos Fundamentales; además incorpora un auténtico sistema organizativo, político y económico que se regula por medio de una compleja distribución de competencias entre los Estados y la Unión. Expuesto de otro modo: el rasgo constitución-declaración se acentúa; se trata de disimular el de constitución-contrato. El primero es aceptado más pacíficamente; el segundo es mucho más discutido. La razón de esta diferencia reside, a mi juicio, en que para muchos existe otra «constitución-contrato», previa al Tratado-Constitución europea, que habría sido firmada por los ciudadanos pertenecientes a cada uno de los territorios demarcados por los Estados-nación. Así, se aprecia que la cuestión no es meramente nominal, tal y como parecía al principio de estas líneas; del uso del término «Constitución europea» - término éste impregnado de los conceptos jurídicos que se utilizaban en relación con el Estado-nación- se desprende un corolario: la discusión sobre la primacía del Tratado-Constitución europea respecto al Derecho doméstico en general y a las distintas constituciones en particular. Pero ésta es una discusión antigua: no se trata de un choque de constituciones sino de regular las relaciones entre Derecho comunitario y doméstico. Mas pensamos que es necesario al menos hacer unas reflexiones al respecto.

La primacía del Derecho comunitario es clara y aceptada en general por casi to$\operatorname{dos}^{8}$. Sin embargo, en 1972, a raíz de la ratificación por el Reino Unido de la European

${ }^{6}$ FRANKENBERG, op. cit., p. 83.

7 Las declaraciones de la por entonces Presidenta del Consejo Europeo pueden verse en http://www. europarl.europa.eu/sides/getDoc.do?language=ES\&type=IM-PRESS\&reference=20070208IPR02888, visitado el 6 de abril de 2008.

8 En concreto, como es sabido, el Tribunal Constitucional Federal Alemán se pronunció contra la incorporación de Alemania al Tratado de Maastricht, principalmente por conculcar el art. 38.1 de la Constitución 
Communities Act, se produjo una polémica interesante. Sir W. WADE defendió que ésta suponía una auténtica revolución, pues modificaba lo que en términos hartianos se llama «regla de reconocimiento». T. AlLAN, en cambio, mantuvo la tesis contraria. No queremos detenernos aquí en esta discusión. Esto ya lo ha hecho, con el detenimiento que se merece, N. MACCORMICK. Éste niega la revolución y afirma que entonces se asistió a una simple modificación en los criterios que incluye toda regla de reconocimiento 9 .

¿Nos podríamos preguntar, por tanto, si al igual que sucedió en 1972 en el Reino Unido, ahora, en toda Europa, se podría producir una revolución si la Constitución hubiera sido aprobada ${ }^{10}$ ? ¿Hasta qué punto se puede hablar de supremacía o de pérdida de soberanía con la llegada de la Constitución europea?

La pregunta, que podría ser superflua si se aborda desde los criterios del Derecho positivo y el nuevo Tratado de Lisboa, posee, sin embargo, en relación con otros aspectos teóricos atinentes a nuestra comprensión de los ordenamientos jurídicos en la actualidad, una enorme virtualidad, pues, a su través, se afrontan problemas propios de la teoría del Derecho. De forma un tanto provocativa y tremendamente lúcida, MACCORMiCK se dirige al núcleo del problema a través del siguiente símil:

«The key question becomes whether there can be a loss of sovereignty at one level without its inevitable and resultant recreation at another. Is sovereignty like property, which can be given up only when another person gains it? Or should we think of it more like virginity, something that can be lost by one without another's gaining it-and whose loss in apt circumstances can even be a matter of celebration? ${ }^{11}$.

Expresado de otro modo: en la Unión Europea nos encontramos — como ha insistido HABERMAS - ante una «constelación posnacional» ${ }^{12}$ para la que ya no es válido el concepto hobbesiano de soberanía. En el seno de la Unión Europea es necesario re-definir el concepto de soberanía, que no puede ser planteado como un juego de suma cero, sino, más bien, como una re-elaboración constante del principio de subsidiariedad, que no debería, al menos en la teoría, conducir a una competición por la soberanía.

Estas afirmaciones son la conclusión (la hemos expuesto con carácter previo, por razones de discurso, no por motivos lógicos, obviamente) de un presupuesto fundamental: tratar de comprender las relaciones entre los ordenamientos jurídicos de los

alemana. Vid. BVerfG, 2 BvR 1877/97 vom 31.3.1998, Absatz-Nr.(1-101), http://www.bverfg.de/entscheidungen/rs19980331_2bvr187797.html, visitado el 20 de marzo de 2008.

9 Vid., en este sentido, N. MACCORMicK, Questioning Soveregnty, Law, State, and Nation in the European Commonwealth, Oxford Univ. Press, 1999, en concreto el capítulo 6 «A Very British Revolution?», pp. 79 y ss. «The constitutional changes that were made in 1972 are capable of being read as changes that envolved the use "of the power of change" to read a new criterion of recognition to the rule of recognition», cita en p. 94.

${ }^{10}$ Hay que tener en cuenta, en este caso, las peculiaridades del sistema de Derecho anglosajón en relación con el continental para que la comparación pueda ser, en algún aspecto, fructífera y correctamente enfocada.

${ }_{11}$ N. MACCORMICK, Questioning Sovereignty. Law, State, and Nation in the European Commonwealth, cit., p. 126.

12 J. Habermas, Die postnationale Konstellation, Suhrkamp, 1998, pp. 91 y ss. Habermas no niega el monopolio de la fuerza ni la soberanía del Estado que, desde un punto de vista formal, permanezcan intactos («formal intakt geblieben sind», p. 107); más bien, sitúa en un primer plano la interdependencia de la política entre los distintos Estados-nación e incide en la necesidad de mantener los logros del Estado-social de Derecho en una sociedad globalizada. 
Estados miembros y de la propia Unión Europea no á la KELSEN —un KELSEN, ciertamente, simplificado-, como un sistema de Stufenbau, sino a partir de las premisas del pluralismo jurídico y asumiendo el riesgo de conflictos o choques entre los distintos ordenamientos. Ésta es la posición defendida por MACCORMICK. No quisiéramos repetir aquí las argumentaciones con las que este autor desarrolla este tema. Mas quisiéramos añadir algunas aclaraciones:

1. El Tratado-Constitución europea, a pesar de la primacía del Derecho comunitario sobre el nacional, no creemos que deba ser interpretada como la cúspide de un zigurat normativo que encadena sus distintos niveles por medio de un mero criterio de jerarquía vertical. El Derecho de la época de la globalización se deja explicar con muchos déficits por medio de la imagen de una cascada normativa, que comience con el Derecho internacional, continúe con el Derecho comunitario y concluya en el Derecho doméstico. En este sentido, precisamente, es interesante la diferencia a la que ha aludido nuestro Tribunal Constitucional entre «primacía» y «jerarquía» ${ }^{13}$.

2. Las relaciones entre Derecho comunitario y Derecho doméstico y, de forma más concreta, entre Tratado-Constitución europea y constituciones domésticas, han de ser analizadas de modo casuístico. Puede ser que, en algunos países, la hipotética aceptación del Tratado-Constitución europea, hubiera supuesto una auténtica modificación de la regla de reconocimiento y no simplemente una añadidura a criterios de esta regla. No se puede hacer una afirmación de carácter completamente general.

3. No obstante, y como consecuencia de la existencia, a un mismo tiempo, de distintos ordenamientos jurídicos interrelacionados, hay que — creemos - aceptar que los «choques» entre éstos no siempre han de tener una solución de carácter jurídico. Habrá que apelar a otros sistemas o subsistemas relacionados con el jurídico para solventar estos problemas; en concreto, al sistema político. Hay que admitir, pues, que los conflictos jurídicos, en algunas ocasiones, si entendemos la soberanía como «virginidad y no como propiedad», deben ser resueltos en instancias políticas y no jurídicas. Esto no tiene nada de negativo si se asume, como propone MACCORMICK, el principio pacta sunt servanda y, además, el sistema político goza de cierta legitimidad ${ }^{14}$. Mas, desde luego, se pone en evidencia algunos de los rasgos clásicos de los ordenamientos jurídicos: completitud y unidad, principalmente. En este sentido, tiene razón MANGAS MARTín cuando afirma que «los Tratados comunitarios ni serán supra-constitucionales, en todo caso son y seguirán siendo extra o meta-constitucionales» ${ }^{15}$.

13 El Tribunal Constitucional ha perfilado la diferencia entre «supremacía» y «jerarquía»: «Primacía y supremacía son categorías que se desenvuelven en órdenes diferenciados. Aquella, en el de la aplicación de normas válidas; ésta, en el de los procedimientos de normación. La supremacía se sustenta en el carácter jerárquico superior de una norma y, por ello, es fuente de validez de las que le están infraordenadas, con la consecuencia, pues, de la invalidez de éstas si contravienen lo dispuesto imperativamente en aquélla. La primacía, en cambio, no se sustenta necesariamente en la jerarquía, sino en la distinción entre ámbitos de aplicación de diferentes normas, en principio válidas, de las cuales, sin embargo, una o unas de ellas tienen capacidad de desplazar a otras en virtud de su aplicación preferente o prevalente debida a diferentes razones». Declaración del Tribunal Constitucional acerca de la existencia o inexistencia de contradicción entre la Constitución Española y la Europea de 13 de diciembre de 2004. Vid. http://www.boe.es/g/es/bases_datos_tc/doc.php?coleccion=tc\&id =DECLARACION-2004-0001, visitado el 20 de marzo de 2008.

${ }^{14}$ Con la expresión «cierta legitimidad» queremos hacer notar que las exigencias y los mecanismos de legitimidad del Estado-nación no pueden ser traspasadas tal cual a las estructuras posnacionales.

15 A. Mangas Martín, La Constitución europea, cit., p. 168. Esta afirmación nos parece muy ajustada. Ahora bien, creemos que en este trabajo, serio y riguroso, se respira, en lo que se refiere al diseño del ordena- 
Podríamos concluir, pues, con una de las reflexiones que A. FisCHER-LESCANO y G. TEUBNER comienzan su último libro, Regime-Kollisionen. Los internacionalistas se imaginan el Derecho internacional dotado de una unidad, a partir de una jerarquía normativa muy similar a la que se creía que existía en el XIX en el Estado Nación. Creen en la centralización del Derecho y les crea profundos problemas que existan distintos centros de producción normativa y distintas instancias de decisión. Poseen una Zentralisierungsvorstellung que no parecen dispuestos a abandonar ${ }^{16}$. En definitiva, TEUBNER y FISCHER-LESCANO afirman menos literariamente lo que afirma MACCORMICK al hablar de las posibles colisiones entre las constituciones de los distintos Estados-Nación y del Tratado-constitucional europeo:

«La fragmentación jurídica del Derecho mundial no se trata ni de una simple colisión de normas jurídicas ni de conflictos sociales, que puedan ser interpretados de modo monocasual. Más bien son contradicciones entre racionalidades institucionales sociales, en proceso de formación, que, con seguridad, no pueden ser resueltas por el Derecho, pero que le exigen un nuevo modo de proceder con sus colisiones normativas» ${ }^{17}$.

\subsection{La tesis del «no demos»}

No sólo los internacionalistas han puesto en duda el carácter constitucional de la «Constitución europea». Los constitucionalistas, politólogos y filósofos del Derecho han ofrecido también aceradas críticas. La más repetida trata de sustentarse por medio de la que se ha llamado la tesis del «no-demos». En realidad, esta tesis es la que subyace (entre otras razones de carácter político, por supuesto) tras el cambio de denominación recogido en el Tratado de Lisboa. Es interesante, por tanto, detenerse en ella.

Según estos argumentos, la sociedad es completamente independiente de cualquier construcción de carácter político o jurídico. El demos radica en la existencia de una «unidad cultural». Esta tesis, que es identificable con la forma de pensar el Estado-nación de las sociedades pre-modernas, niega la existencia de un «pueblo europeo»y, consecuentemente, la posibilidad de una Constitución europea. Tal vez su elaboración más acabada se encuentre en los trabajos de D. GRIMM. Ya en 1994 publicó un extenso artículo en donde se cuestionaba la necesidad de una Constitución europea: «Necesita Europa una Constitución» ${ }^{18}$. En 1999 insiste sobre el tema. Publica un artículo de opinión para el semanario alemán Die Zeit ${ }^{19}$, cuyo subtítulo condensa bien su contenido: «Eine demokratische EU braucht bessere Institutionen, aber kein Grundgesetz» (una Unión Europea democrática necesita mejores instituciones, pero no una Constitución). Más recientemente, en 2004, dictó una conferencia en el Walter

\footnotetext{
miento jurídico internacional, cierto aire kelseniano, así, por ejemplo: «Si la "Constitución" europea es explícitamente una norma de rango superior a las Constituciones nacionales, ¿¿su evolución y sus revisiones ya no tiene que contrastarse con las Constituciones nacionales?» (p. 172). Las cursivas son propias.

16 A. Fischer-LesCANo, Regime-Kollisionen. Zur Fragmentierung des globales Rechts, Suhrkamp, 2006, pp. 10 y ss.

17 A. FISCHER-LESCANO, op. cit., p. 24

18 D. GRIMm, Braucht Europa eine Verfassung?, Carl Friedrich von Siemens Stiftung, 1994.

19 D. GRIMM, «Ohne Volk keine Verfassung. Eine demokratische EU braucht bessere Institutionen, aber kein Grundgesetz», vid. http://www.zeit.de/archiv/1999/12/199912.verfassung_xml, visitado el 16 de enero de 2008 .
} 
Hallstein-Institut para Derecho constitucional europeo con el título «Integration durch Verfassung». Es interesante observar cómo los énfasis de la argumentación se desplazan de unas ideas a otras.

La argumentación de GRIMM se fundamenta en un conjunto de premisas que le conducen a negar la necesidad de una Constitución europea:

1. La inexistencia del sujeto del proceso democrático.

2. La relación entre integración y Constitución.

3. La no implicación directa entre Democracia y Constitución.

Ad 1) Existe una delimitación previa del concepto de Constitución con el que se debe contrastar la hipotética y futura «Constitución europea». Un concepto construido históricamente ${ }^{20}$, y que se puede delimitar con lo que PRIETO SANCHís y otros autores han llamado «constitucionalismo moderno»; entendiendo éste no como un punto estático de llegada sino como un proceso ${ }^{21}$. La «Constitución europea» tendría que ser analizada en el marco de las constituciones que nacen a partir del XVIII, y que GRIMM caracteriza con el adjetivo «normativo». La Constitución americana y la francesa funcionarían como archivo, entendiendo este término a lo DERRIDA, de cualquier Constitución $^{22}$.

Normatividad, pero también consenso. Las constituciones modernas se caracterizarían por garantizar la convivencia de un conjunto de ciudadanos libres sin utilizar para su legitimación argumentos de carácter teológico. En el plano del discurso teórico, el giro de legitimación se habría producido en el iusnaturalismo ilustrado; en el terreno de la práctica, en la Revolución Americana contra el parlamentarismo inglés y en la Revolución Francesa contra el absolutismo. Consenso inmanente que a su vez produce (al menos así lo afirma por ahora GRIMM) un alto grado de integración social $^{23}$. Esta afirmación, sin embargo, debe ser matizada en los términos expuestos por PRIETO SANCHÍs ${ }^{24}$.

20 H. Mohnhaupt, «Verfassung I und Grimm, D., Verfassung II», en Brunner, Conze y Koselleck (Hrsg.), Geschichtliche Grundbegriffe, Stuttgart, Band \&, 1990, pp. 861 y ss.

21 PRIETO SANCHÍs lo ha definido del siguiente modo: «Con independencia de que pueda hablarse de un constitucionalismo antiguo y medieval, el constitucionalismo moderno es aquel proceso histórico cultural en virtud del cual la relación entre los detentadores del poder y quienes están sujetos al mismo se configura como una relación jurídica, definida, regulada y sometida a reglas jurídicas conocidas; y este proceso emerge en un cierto contexto temporal». L. PRIETO SANCHís, Justicia constitucional y derechos fundamentales, Madrid, Trotta, 2003 , p. 35.

22 Sería interesante repensar el término «Constitución» a partir del análisis que DERRIDA hace de la expresión archivo. Al menos las notas de «origen», «mandato» y rearticulación entre lo privado y lo público se encuentran unidas al concepto de «Constitución». Al mismo tiempo, las «instrucciones para la interpretación» son parte fundamental de todo archivo, el diseño de una casta de archiveros (de arcontes) es consustancial, del mismo modo, a la idea de «Constitución». J. DerRIDA, Mal de archivo, Trotta, 2006.

23 D. Grimm, Braucht..., cit., p. 24.

24 De nuevo quisiéramos citar el excelente trabajo de PRIETO SANCHís: «Como ya advirtiese JELLINEK hace más de un siglo, el contenido normativo de los derechos del constitucionalismo americano — por ejemplo del Bills of Right de Virginia - procedía también de la tradición inglesa y su formulación presentaba una fuerte impronta religiosa (...)». L. PRIETO SANCHÍs, Justicia constitucional y derechos fundamentales, p. 42. En general, se muestra como el iusnaturalismo racionalista — a pesar de su mala prensa - no es en absoluto ajeno al proceso constitucional. Como se advierte en este trabajo el positivismo también habría desempeñado un papel importante en el constitucionalismo moderno, a saber: la teoría de la interpretación positivista habría sido crucial para el control de la ley (p. 93). 
¿Los argumentos expuestos no refutarían la posibilidad de una Constitución europea? Sólo hasta cierto punto, incluso GRIMM sostiene que existe un grado de constitucionalidad en los Tratados europeos, pues con la formación de la Unión Europea, desde un punto de vista jurídico, se está creando una alta autoridad, cuyas competencias no tienen que ser en cada acto jurídico ratificadas, como si se tratase de un Tratado internacional, por los firmantes.

Pues bien, este tipo de constituciones normativas se caracterizan porque el sujeto que las impulsa es un «pueblo» que, en teoría, estaría unido por lazos de carácter social, cultural o histórico. Primero existe el pueblo, luego la Constitución. Por tanto, la inexistencia de un «pueblo» europeo sería, a juicio de los defensores de esta tesis, entre los que se encuentra GRIMM, uno de los impedimentos fundamentales que cierran el paso a una Constitución europea.

La tesis podría, muy resumidamente, enunciarse por medio de la siguiente cita:

«Una Constitución, en el sentido completo de la palabra, requiere de un acto inicial que dependa del pueblo o que, al menos, demuestre la capacidad política de éste. Una fuente de este tipo falta en el Derecho primario comunitario. No se retrotrae a un pueblo europeo sino a cada uno de los Estados Miembros y permanece dependiente de éstos» ${ }^{25}$.

Hoy en día este argumento, que apela al «pueblo» — cargando esta palabra de sentido cultural o étnico- como sujeto estático y necesario de todo proceso constitucional ha devenido obsoleto e insostenible ${ }^{26}$. Principalmente por dos razones:

En primer lugar, el ya citado art. I-1 del malogrado Tratado por el que se establecía una Constitución para Europa y que sitúa en condiciones de paridad a los Estados miembros y a los ciudadanos europeos. Y, en segundo lugar, se debe hacer notar que la suspensión temporal del proyecto constitucional europeo se produce, precisamente, por el rechazo de los ciudadanos que le han retirado su apoyo; no por los altos mandatarios. Tal vez esto sea muestra (seamos optimistas e ingenuos) de que los procesos de europeización no se están produciendo al margen de la opinión pública europea ${ }^{27} \mathrm{o}$, dicho de otro modo, que si tratan de ejecutarse en espacios opacos a la opinión pública terminan siendo paralizados o, al menos, ralentizados ${ }^{28}$.

25 D. GRIMM., Braucht, p. 31.

26 En el mismo momento que se elige el Parlamento Europeo nacería el pueblo europeo. En este sentido, el concepto de demos no es anterior al de Constitución sino simultáneo. La voluntad colectiva de crear un cuerpo político constituiría al propio pueblo. Sólo, por tanto, las instituciones democráticas son las que podrían facilitar el paso de la sociedad al demos. Esta construcción podría ser utilizada tanto para el Estado-Nación como para las estructuras postnacionales como la Unión Europea. Bajo ella subyace un concepto de pueblo meramente jurídico (no político ni sociológico). Tal vez sea éste el punto de arranque más adecuado de cara a constituir una sociedad democrática, pero hay quienes lo critican por su formalismo. Es necesario que exista una sociedad que esté relativamente cohesionada (no tiene que existir una homogeneidad religiosa, ni de valores culturales) para que, a partir de ella, se puede crear un demos meramente jurídico.

27 No creo que sea correcto argumentar que en el caso francés u holandés el rechazo de la «Constitución europea» fuera un voto de castigo a la mala gestión de las políticas domésticas. Parecería que los ciudadanos no supieran realmente lo que están votando. Más aún en el caso de Holanda, en donde el referéndum no era obligatorio.

${ }_{28}$ Desoladores son los datos ofrecidos por el CIS en su encuesta sobre El XX aniversario del ingreso de España en la Unión Europea (Cuestionario 2641) http://www.cis.es/cis/opencms/-Archivos/Marginales/2640_2659/2641/e264100.html, visitado el 20 de marzo de 2008. Así un 49,7 por 100 se considera poco informado y un 19,7 por 100 nada informado sobre los asuntos de la UE. Un 35,7 por 100 dice que no «ha oído 
Ad 2) Si bien la realidad y la evolución de los acontecimientos han mostrado que el argumento del «no demos» no pierde predicamento, de todos modos, los autores que lo defienden han tratado de reestructurar su discurso, manteniendo, sin embargo, sus posiciones fundamentales.

Así, en primer lugar, el argumento del sujeto se reelabora, ya no se defiende la idea de un pueblo constituido por una serie de tradiciones comunes; ahora se enuncia la idea de un pueblo realmente europeo. Los ciudadanos europeos no estarían participando en el debate por ser «ciudadanos de la Unión»; lo harían en calidad de «ciudadanos de un Estado miembro». De este modo, en buena medida, se trata de solventar de este modo las dificultades a las que se aludían anteriormente (el artículo primero del Tratado y el rechazo de la Constitución por los ciudadanos franceses y holandeses) ${ }^{29}$.

En segundo lugar, se enfatiza sobre los argumentos de carácter cultural, para ello se emplea la idea de «integración». Se comienza mostrando las dudas de que las constituciones nacionales produzcan algún tipo de integración entre los ciudadanos. Más bien, la integración no sería un efecto de las constituciones; se constituye en su punto de partida. Los diversos factores que pueden cohesionar una sociedad son de carácter fáctico y pre-existentes a las propias constituciones: el concepto de nación, el de homogeneidad cultural. Estos constituirían la identidad colectiva, una comunidad, un sujeto colectivo a partir del cual se fundarían las constituciones como posibilidad de regular la convivencia. Éste podría ser el primer paso hacia un nacionalismo étnico-cultural que, como es sabido, nos ha llevado hacia un terreno muy resbaladizo, deslizándose progresivamente hacía un nacionalismo puramente étnico. La conclusión aplicada a la Unión Europea, puede ser expresada en un doble sentido:

De una parte, sólo aquellos países miembros de la Unión Europea que presentan una «integración previa» de este tipo pueden formar parte de ella ${ }^{30}$. En segundo lugar, debido al «nivel de integración» que existe en la Unión Europea (que sería, obviamente, un nivel bajo) no es necesario arbitrar una Constitución sino mecanismos, por así decir, más débiles. No es necesaria una Constitución sino un Tratado; no es necesaria una carta de Derechos fundamentales, basta simplemente la jurisprudencia del Tribunal Europeo de Derechos Humanos. Y, por último, pero no menos importante, la Constitución europea carecería de un auténtico «constitutional moment», pues se presenta como un simple jalón más en la evolución de la Unión Europea. Este argumento ha sido defendido por autores de gran solvencia. Según, por ejemplo, HABERMAS,

\footnotetext{
hablar del Consejo de Ministros»; la misma afirmación la suscriben respecto a la Comisión europea el 34,2 por 100 y respecto al Parlamento Europeo el 28,2 por 100 de los entrevistados.

29 D. GRIMm, Integration durch Verfassung, Vortrag an der Humboldt Universität am 12. Juli 2004, p. 15.

30 Esto no siempre ha sido así. Antes se prestaba mayor atención a los parámetros de carácter económico; se trataba de formar un «mercado económico europeo». Ahora, sin embargo, como se aprecia en relación con los nuevos países miembros, se presta, al menos en teoría, más atención a la ausencia de corrupción y al funcionamiento del sistema judicial. El debate sobre si países como Turquía están preparados para su integración en la Unión Europea ha mutado de los datos económicos a una discusión, a veces no carente de cinismo, sobre si éste país respeta más o menos los Derechos humanos. No obstante, un análisis serio sobre la buena salud de las estructuras jurídicas y sociales y la ausencia de corrupción hubiera sido necesario en todos los casos. Éste fue un error que se cometió, a nuestro juicio, con países como España y Portugal. Vid., al respecto, el artículo de P. PINZLER en Die Zeit, «Bis sie irgendwann platz», núm. 48, de 28 de septiembre de 2006, p. 10.
} 
las constituciones son hijas de revoluciones o de momentos de crisis; sin embargo, la Constitución europea no se podría incardinar en este modelo explicativo. Parece claro que el intento de ratificar el Tratado por el que se aprobaba una Constitución europea ha llegado sin revoluciones y en una época de paz. La idea de refundación o de la necesidad de que la Unión Europea se una a un «transfer» en el Derecho subyace tras esta tesis, que, históricamente, tiene su mirada puesta en los procesos constitucionales americano y francés. Es curioso, por tanto, que el propio HABERMAS insista (criticando a L. SIEDENTOP ${ }^{31}$ ) sobre lo poco que hay en común entre el modelo americano constitucionalista y el europeo de nuestros días: y ello, porque las circunstancias históricas sociales y económicas son completamente distintas y defienda, a la par, este argumento. Además, esta teoría del «constitutional moment», también defendida por D. GRIMM, elude que los intentos de construir algo así como «una Constitución» han sido consustanciales al proyecto europeo desde sus inicios, y no pueden ser fijados, como usualmente se hace, al discurso de J. FisCHER en la Humboldt. Precisamente, el inicio del proyecto europeo está circundado por duros momentos de crisis; hay que recordar que Europa renace justamente tras las dos Guerras Mundiales y se afianza cuando se retira el telón de acero. Como ya se ha apuntado, la incorporación de muchos Estados Miembros a este proyecto puede ser tan sólo interpretada adecuadamente en el marco de las salidas de las dictaduras y como un modo de exorcizarlas (España, Portugal y países del este, principalmente).

Ad 3) Democracia y Constitución. Sorprendente parece, en un primer momento, la afirmación de J. HABERMAS en uno de sus trabajos más conocidos y re-editados, Warum Europa braucht eine Verfassung?

«... la cuestión constitucional no proporciona la llave para el problema principal que tenemos que resolver. Si atendemos a la trasformación que nos antecede, no se trata de inventar nada, sino de conservar los grandes logros democráticos de los Estadosnación europeos, más allá de sus propios límites» ${ }^{32}$.

Dicho de otro modo, el Tratado-Constitución europea sería una «condición necesaria pero no suficiente» para defender el modelo de Estado social posbélico ${ }^{33}$. El relativo escepticismo de HABERMAS está orientado a analizar un lazo que, desde hace tiempo, parece consistente, el de Constitución/democracia. Cualquier Constitución no supone, a su juicio, automáticamente un incremento de la democracia. Y así sucede (por mucho que nos pese) con el Tratado-Constitución europea. Es cierto; afirmar otra cosa sería una ingenuidad. Según HABERMAS, la «Constitución europea» no funcionaría (se podría expresar de este modo) en el mero terreno de lo jurídico; debe constituirse en un importantísimo catalizador para azuzar la opinión pública, para crear estructuras de opinión pública que, a la postre, son las que han ofrecido un suelo estable a los procesos jurídicos europeos.

Ahora bien, no creemos —y enseguida ofreceremos algún ejemplo— que el Derecho comunitario funcione sólo en la esfera de la opinión pública, también actúa,

31 L. SiEdEnTOP, La democracia en Europa, Madrid, Siglo XXI, 2001.

32 J. HABERMAS, «¿Por qué Europa necesita una Constitución?», en New Left Rewiew, p. 6. Se puede consultar en http://www.newleftreview.info/PDFarticles/Spanish/NLR24501.pdf, visitado en enero de 2008.

33 J. HABERMAS, «¿Por qué...?», cit, p. 12. 
obviamente, en el campo jurídico, para bien y para mal. He aquí el ejemplo: N. MACCormick empieza uno de sus últimos libros, Who's Afraid the European Constitution $^{34}$, por el núcleo de la cuestión constitucional. Desde el 1 de enero de 2004 el Reino Unido ha reconocido y está utilizando el «European Arrest Warrant» (en adelante, EAW) que, como es sabido, se ha articulado a través de la Parte I del Tratado de Extradición Europea. Por medio de este mecanismo jurídico, una orden de arresto de un país de la Unión Europea puede ser llevada a cabo por una autoridad judicial de un tercer país. Llamativo que uno de los argumentos empleado por los británicos contra el EAW es que podría conculcar las tradiciones democráticas británicas tan respetuosas con los derechos fundamentales; en concreto, MACCORMICK se refiere al Habeas Corpus inglés y a la regla de los «bundred and ten days» escocesa. Esta falta de «democracia» de EAW vendría causada, a juicio de este autor, porque no es el Parlamento Europeo sino el Consejo de la Unión el que la estableció. Aunque la argumentación no carece de razones sólidas, en algunos aspectos no es del todo coherente; sobre todo en el caso del Reino Unido, pues, desde la Terrorism Act ${ }^{35}$, se eliminó para todo el territorio del Reino Británico la exigencia del Warrant y del $\mathrm{Ha}$ beas Corpus: entonces, ¿cómo exigir a los demás una «tradición» que ellos mismo no respetan? Esto ha hecho, que bajo la sombra de Churchil, se pueda llegar a detener a alguien por hacer una manifestación, disfrazada de picnic (Serious Organised Crime and Police Act de 2005) ${ }^{36}$.

La Constitución europea y, en general, un Derecho europeo, creado al abrigo de los derechos humanos, conllevan, en principio, un mayor respeto a la democracia. En este sentido, es interesante la propuesta (desgraciadamente poco exitosa) del propio MACCORMicK de crear un habeas corpus europeo. Las tentaciones del Estado-nación de limitar los derechos clásicos son cada vez más fuertes. La creación de «tradiciones» y la evocación del viejo «espíritu democrático» no eliminan per se el peligro de conculcar los derechos humanos. Más bien parece que es justo al revés. Sin embargo, un Tratado-Constitución europea, que clarifique y fije cuáles son los derechos que deben ser respetados por todos los Estados-nación y reconocidos a todos los ciudadanos, puede ser una herramienta jurídica importante más allá de las veleidades e irracionalidades de los Estados miembros. El principio de subsidiariedad, por tanto, se debería dejar para los calabacines y las pescadillas ${ }^{37}$, pero no para los derechos humanos ${ }^{38}$.

Es posible que el Tratado-Constitución europea conllevará un incremento de la democracia en las instituciones europeas. El optimismo de MACCORMICK parece fundado; ciertamente las reformas del Parlamento Europeo incrementarían la democra-

${ }^{34}$ N. MACCormick, Who's Afraid of a European Constitution?, Imprint Academic, 2005, vid. el primer capítulo.

${ }^{35}$ «Act Terrorism, Art 41. (1) A constable may arrest without a warrant a person whom he reasonably suspects to be a terrorist», http://www.opsi.gov.uk/acts/acts2000/20000011.htm, visitada el 20 de marzo de 2006.

36 Sobre este hecho, léase el artículo de H. PORTER, «Blair laid bare: the article that may get you arrested», en The Independent, 29 de junio de 2006.

37 Tomamos la expresión prestada de MACCORMICK quien se queja de la inclusión de la política de pesca dentro de la agraria, por lo que afirma que los «calabacines no son lo mismo que las pescadillas».

38 El problema fundamental es qué mecanismos están en la mano de la Unión Europea para garantizar los Derechos Humanos y si realmente existe voluntad política para algo más que declaraciones formales, tamizadas por el principio de subsidiariedad. 
tización en el seno de la Unión europea ${ }^{39}$. Ahora bien, a nuestro juicio, no lleva razón al afirmar que pocos procesos constituyentes han disfrutado de un proceso tan democrático, tan abierto y visible. Sobre todo, si pensamos como se ha maniobrado a partir de la negativa holandesa y francesa, hasta culminar en el Tratado de Lisboa. Lo cierto es que muchos ciudadanos no se ha enterado de nada. Según una encuesta publicada en el Frankfurter Allgemeine Zeitung realizada, entre marzo y abril del 2003, a 16.000 ciudadanos europeos, el 57 por 100 de los encuestados desconocía completamente la existencia de la Convención para la redacción de una Constitución europea ${ }^{40}$. A lo peor tenía razón el informe de G. STUART: el consenso parece haberse diluido en un mero acuerdo de elites.

No obstante, la cita de HABERMAS — ofrecida anteriormente — señala una reflexión de más calado. Lo esencial no es la construcción de un «instrumento jurídico» de una enorme complejidad (que, a nuestro juicio, no puede ser encorsetado en el concepto «Tratado» o «Constitución»), lo que se le antoja al autor alemán fundamental es el «peso simbólico» del Tratado-Constitución. ¡No es de extrañar! HaBERMAS advierte que la Constitución europea (él emplea aún este término), a pesar de sus múltiples defectos (sobre todo, a nuestro juicio, de la poca importancia concedida a los derechos sociales) ha empezado, aun estando herida de muerte -o precisamente por ello- a modificar el espacio público europeo, a suscitar debates, a inspirar líneas editoriales: el proceso por el que se ha llegado al Tratado de Lisboa modifica las estructuras de la opinión pública europea, y lo hace precisamente europeizándolas. Adquiere, pues, sentido afirmar que las preguntas iusfilosóficas, constitucionales o de Derecho comunitario de carácter quasi-metafísico aligeren su importancia; los caminos parecen otros. El procedimiento, la discusión y la crisis de la Constitución europea —o como se la quiera llamar- son un acicate fundamental para el fortalecimiento de un espacio público europeo; este mensaje debe ser sacado de la botella y puesto en algún lugar visible.

Ciertamente, esta idea requiere de algunas puntualizaciones. Quisiéramos mencionar, al menos, dos.

1. Las modificaciones de la estructura pública, del espacio público y su relación con la democracia han sido objeto de estudio en un conocido trabajo de HABERMAS ${ }^{41}$. Como es lógico, todo lo expuesto allí, no puede ser traído sin más al espacio europeo. Los cafés y casas de té se han convertido, en muchos casos, en hamburgueserías, o bien han desaparecido. La democracia en el Estado-nación se amparó en la creación de espacios públicos abiertos a la discusión. La Europa que anhelamos, por tanto, debe seguir el mismo camino. Esta regla de tres no funciona: los periódicos no se escriben en europeo ni las cadenas de televisión son iguales en toda Europa. Hay excepciones ${ }^{42}$. El espacio europeo es mucho más difuso, heterogéneo y débil que aquel del que disfrutó el Estado-nación. Los mecanismos que lo pueden fortalecer, aún estando ahí (Internet,

39 N. MACCORMICK, Who's Afraid of a European Constitution?, cit, p. 50. El Tratado de Lisboa incorpora un nuevo Título II con el rótulo de «disposiciones sobre los principios democráticos» por el que se pretende dar más peso al Parlamento.

${ }^{40}$ Citado por BECK y GRAndE en su libro Das Kosmopolitische Europa, cit., p. 343.

41 J. HABERMAS, Strukturwandel der Öffentlichkeit, Suhrkamp, 1990.

42 La revista Lettre internationale se publica, con distintos contenidos, en distintos idiomas. Le monde diplomatique o la cadena de televisión francesa Arte también poseen una amplia cobertura en Europa. 
televisión por cable, política lingüística, etc.), no terminan de consolidarse en todas las capas sociales europeas. Esto, hasta cierto punto, no es un problema. Europa no puede mirarse constantemente en el espejo del Estado-nación, pues el hechizo surtiría menos efecto que en los cuentos infantiles al uso. Europa ha de aceptarse a sí misma y reconocer que sus estructuras, tanto jurídicas como sociales, no son idénticas a las del Estado-nación. Tampoco es necesario. HABERMAS ha puesto de manifiesto qué debe significar este espacio público europeo ${ }^{43}$.

2. No sólo se trata de «mantener las conquistas realizadas por el Estado social de Derecho» en el marco del Estado-nación, también se desea incrementarlas. La construcción europea nace con otras finalidades y, sobre todo, ha creado problemas que antes, en el Estado-social del periodo de posguerra, al menos no existían en las dimensiones y formas que ahora han adoptado. Sólo a modo de ejemplo quisiera señalar la aparición de nuevas fronteras con la creación de la Unión europea. Éstas no sólo la delimitan hacia el exterior, sino fronteras que, a pesar de la cacareada libertad de movimiento, se han creado en su interior, ya sea por mecanismos jurídicos o sociales. Programas informáticos en los servicios tributarios que no aceptan el número de pasaporte del ciudadano europeo y que le obligan a solicitar una tarjeta de extranjero para poder tributar por su actividad profesional; la obligación de empadronamiento en muchos países europeos (por ejemplo, en Austria), demostrando que se poseen bienes suficientes o los problemas para abrir una cuenta bancaria en el Reino Unido, pues es necesario tener una residencia fija, que debe ser demostrada por el pago de las «utilities» son algunos ejemplos (quizá triviales) de los obstáculos que se encuentra cualquier persona al moverse dentro de la Unión Europea. Si la capacidad económica disminuye, se incrementan las fronteras. Fronteras simbólicas (esto no es nuevo) que unen, pero separando, como burbujas de cristal. El desplazamiento desde Rumanía o Polonia a las granjas escocesas, a la recogida de la fresa en Huelva o a la campiña austriaca ${ }^{44}$ es sólo una modificación geográfica no social: muchos ciudadanos de la Europa del Este se encuentra, por ahora, paradójicamente, fuera de Europa, y ello a pesar de la integración de sus países. Los guetos en las zonas agrícolas, los contratos de temporada, el uso de los trabajadores como mera mercancía y la «regulación de flujos» migratorios no se limitan al extranjero. Europa tiende a separar y unir al mismo tiempo. Z. BAUMAN, en su maravilloso libro, «Europa una aventura inacabada», lo ha expresado mucho mejor:

«No se puede negar al "otro" puesto que incorpora el fenómeno de la "otredad": en la práctica del europeísmo, el esfuerzo por separar, expulsar y externalizar se ve constantemente socavado por la atracción, admisión, acomodación y asimilación de lo "externo"» ${ }^{45}$.

3. Estos no son nuevos problemas, pero sí nuevas conquistas que se deben hacer desde el espacio europeo y desde el Tratado-Constitución europea ${ }^{46}$; en definitiva,

43 Vid. J. HABERMAS, «¿Por qué...?», cit. p. 18, también del mismo autor Der gespaltenen Westen, p. 80. La idea de HABERMAS es que se deben abrir los espacios nacionales al debate europeo, creando de este modo una dinámica supranacional.

44 Al respecto, la excelente película de R. MADER, Struggel, Austria, 2003.

45 Z. BaUman, Europa. Una aventura inacabada, Losada, 2006, p. 19.

46 Para BAUman el Estado social fue una solución (con un poco de suerte) al problema que planteaba la retirada de Europa de sus posesiones coloniales allende de los mares» (p. 117); sin embargo, hoy, a juicio de este autor, no se puede seguir manteniendo la máxima «soluciones locales para problemas globales». Por esto «un Estado social no se puede construir ni sostener en un Estado territorialmente por sí sólo; tal vez ni siquiera 
habrá que ampliar el círculo de la solidaridad no sólo a los nacionales sino a todos los europeos. Las conquistas de los «grandes momentos legislativos» no se deben diluir en los reglamentos o en las órdenes ministeriales. Aquí el Tratado-Constitución europea puede hacer bien poco; a veces lo simbólico se convierte en pura estética.

Por último, el incremento democrático que pudo haber traído el Tratado-Constitución europea y que pretende traer el Tratado de Lisboa guarda una estrecha relación con la forma en que se pueda organizar el espacio europeo, la estructura europea. ¿Se puede organizar ésta bajo una estructura organizativa ya acuñada en la teoría del Estado?

J. FISCHER en su discurso «Vom Staatenverbund zur Föderation. Gedanken über die Finalität der europäischen Integration ${ }^{47}$ en la Humboldt Universität de Berlín apostó - con las matizaciones ya señaladas- por una Europa federal sui generis. ¿ ¿Se puede hablar de una asociación federal? ¿Qué consecuencias tendría esto para la democracia en Europa? Los nacionalistas o los defensores del Estado-nación a la antigua usanza se muestran reacios con esta idea. MACCORMICK, por ejemplo, prefiere hablar de un sistema de «confederación sui generis». Desde un punto de vista democrático, un sistema con un único centro donde se toman las decisiones implicaría, desde luego, un déficit democrático; y, por tanto, un sistema de esta índole pondría en tela de juicio la soberanía de los distintos Estados nación. No parece lo más adecuado distanciar aún más las instituciones europeas del ciudadano. Los principios de subsidiariedad y proporcionalidad reconocidos y desarrollados en el protocolo anexo al Tratado de Lisboa parecen reforzar la opinión expuesta por MACCORMICK. Una «confederación sui generis» no acabada: el reparto de competencias entre la Unión y los Estados miembros apunta en este sentido.

De nuevo surge el mismo problema al que hacíamos referencia anteriormente: se quieren encapsular en conceptos antiguos realidades nuevas. Además, como han puesto de manifiesto U. BECK y E. GRANDE ${ }^{48}$, se emplea para ello la lógica del «si es esto no»: la Unión Europea si es esto no puede ser aquello. Estos autores sostienen que la Unión Europea ${ }^{49}$ trata de pasar de un juego de suma cero a uno de integración, en donde compaginan tanto las perspectivas de los federalistas como las de los intergobernalistas en una estructura supranacional. Europa no puede construirse a las espaldas del Estado-nación, pero, a la par, está formando un nuevo tipo de estructura.

dentro de una "fortaleza" que incorporase una combinación de varios Estados con la intención de reforzar su defensa» (p. 119). Tanto BAUMAN, como HABERMAS y U. BECK, a pesar de las diferencias que existen en sus planteamientos, apuestan por una Europa cosmopolita á la Kant (por supuesto, más allá del derecho de visita del que habla KanT en la Paz Perpetua).

47 J. FISCHER, «Vom Staatenverbund zur Föderation. Gedanken über die Finalität der europäischen Integration», en W. LOTH, Entwürfe einer Europäische Verfassung. Eine historische Bilanz, cit., pp. 241 y ss.

48 U. BeCK y E. GRANDE, Das kosmopolitische Europa, Suhrkamp, p. 61.

49 BECK y GRANDE diferencia entre Unión Europea y Europa. La diferencia es interesante y, a nuestro juicio, puede arrojar cierta luz sobre algunos problemas espinosos. Hay que tener en cuenta que el libro de BECK y GRANDE afronta, este tema desde la perspectiva sociológica. Pero, aunque aquí utilizamos el término Unión Europea en un sentido normativo-jurídico, habría que preguntarse hasta qué punto éste no es un tanto corto de miras. ¿No ejercerá, como tantas veces ha sucedido, lo fáctico-sociológico una suerte de fuerza de atracción sobre lo normativo? ¿No terminará confundiéndose la Unión Europea, debido a su carácter procesal, con lo que usualmente denominamos Europa? ¿Puede ser Europa/Unión Europea una realidad simplemente geográfica? ¿Puede existir Europa/Unión Europea sin integrar a Rusia, acelerando todo lo posible la democratización de este país? 
Esta tesis, que, debido a su extensión, no podemos comentar ahora detalladamente, se fundamenta en una filosofía de la historia compleja en la que los cambios sociales, políticos y económicos están impregnados de la dialéctica de continuidad y discontinuidad $^{50}$.

La conclusión a la que se puede llegar es que el Tratado de Lisboa y el proceso de constitucionalización del Derecho comunitario en general pueden incrementar la democracia en Europa por el debate que está generando; a juicio de BECK y GRANDE, por varias razones: por introducir nuevas estructuras postnacionales que habrán de coadyuvar a construir el fundamento normativo sobre el que puede nacer la sociedad europea (no al revés), y porque puede institucionalizar un régimen político de cuño cosmopolita en el seno de Europa.

\section{CONSTITUCIÓN Y CIUDADANOS: QUÉ NEXO}

Es posible que los pueblos perciban que su unión depende de sentimientos de carácter irracional. La pertenencia a un pueblo o una nación podría ser explicada por disfrutar de una cultura gastronómica, poseer una «historia común» o un patrimonio musical determinado. Esto puede llevar a lo que se ha llamado «culturalismo esencialista». Sin embargo, en general, los rasgos culturales son malos indicadores de la pertenencia a una nación/pueblo - por emplear los términos clásicos-; en un mundo globalizado como el nuestro la cultura es cada vez más difusa y heterogénea. Pero lo que nos interesa subrayar es que, a nuestro juicio, desde un punto de vista jurídiconormativo, no nos podemos dejar seducir por estos elementos de carácter románticoesencialista, que, a menudo, están basados en un modo muy concreto de interpretar y escribir la historia. Así no queremos negar que exista cierto «pegamento irracional», que une a los grupos, comunidades, ciudades, naciones o estructuras posnacionales — dicho sea de paso que los intentos de construir estas irracionalidades a veces, por su voluntarismo, rozan la hilaridad y la ingenuidad: pensamos, por ejemplo, en el intento de hacer de la Novena Sinfonía de Bethoven el himno europeo-; pero el plano normativo debe ser ciego a este sentimentalismo.

Quisiéramos señalar dos intentos serios de construir un nexo razonable entre ciudadanos y Unión Europea y que, quizá, puedan funcionar como sustento del proceso de constitucionalización del Derecho comunitario; dos construcciones que, curiosamente, han encontrado su banco de pruebas en el Estado-nación, al que muchos quieren oponer estructuras posnacionales como la de la Unión Europea. A nuestro juicio, como ya he expuesto, esto no es un problema. La dialéctica entre Estado-nación y estructuras posnacionales se debe definir por relaciones de continuidad-discontinuidad, evolución/revolución. Ésta forma de pensar, que, como ya se ha señalado, ha sido propuesta por BECK y GRANDE, nos parece mucho más fructífera que un dualismo excluyente. HABERMAS y MACCORMICK se mantienen cercanos a esta idea. Precisamente quisiéramos comentar dos de los argumentos principales de estos autores, que, en realidad, presentan algunas analogías, el Verfassungspatriotismus y el civil nationalism. ¿Hasta qué punto se pueden integrar estas construcciones en Europa como modo de

${ }^{50}$ U. Beck y E. GRANDE, Das kosmopolitische Europa, cit., p. 54. 
crear una cohesión jurídica? ¿Pueden ser un primer paso para la construcción de una cohesión social no esencialista-cultural? ¿Qué problemas — discontinuidades- presenta su aplicación a la Unión europea/Europa, cuando, en un primer momento, se idearon desde el Estado-nación?

\subsection{Patriotismo constitucional}

La idea del «Verfassungspatriotismus» es reivindicada por HABERMAS en la conocida «Historikerstreit»: una disputa contra algunos historiadores (NOLTE, HiLDBAND....) que se produjo por dos razones principalmente: tratar de explicar el «Holocausto» como reacción contra los bolcheviques, y negarle su especificidad moral y reducirla a una de carácter técnico. HABERMAS sostiene, con mucha razón, que este modo de hacer historia es una manipulación ideológica de la historiografía y que no debe ser tolerada como discusión en el espacio público. Las iras habermasianas también se encrespan contra el Frankfurter Allgemeine Zeitung, periódico que publica el artículo de NolLTE. Si hubiera sido en un medio especializado, afirma HABERMAS, no hubiera sido necesario contestar. Esa relación con la esfera pública de la interpretación historiográfica, ideológicamente orientada, parece funcionar como un resorte que hace a HABERMAS, a partir de los argumentos del escrito de JASPERS sobre la culpabilidad, participar en la discusión. Parece, pues, una diatriba en alemán, entre alemanes y para alemanes ${ }^{51}$.

Es precisamente ese miedo a la «tradición delictiva», ese «principio de responsabilidad jurídica colectiva», que se extiende a las generaciones futuras - y sobre el que más tarde HABERMAS vuelve a incidir al escribir el prólogo para el famoso libro de D. GOLDHAGEN, «Los verdugos voluntarios de Hitler» ${ }^{52}$ - el que lo lleva a abrazar el Verfassungspatriotismus de STERnBERGER. Parece, pues, que —y éste sería el primer obstáculo que debe ser superado- esta teoría nace junto a un Estado-nación clásico o, mejor dicho, como la reacción a la forma más exagerada y desproporcionada de un Estado-nación: la étnico-racista. El patriotismo constitucional, en un principio, está pensado para un Estado-nación; se trata de una re-definición del Estado de Derecho que se hace a partir de ciertas vicisitudes históricas a las que éste se debe enfrentar. ¿Se podría «descontextualizar» este término y utilizarlo en el seno de la Constitución europea? La respuesta es, a nuestro juicio, sí. Y lo es por dos razones. En primer lugar, del Holocausto no sólo se desprenden consecuencias de carácter moral o jurídico para Alemania; la imposibilidad de repetición y lo específico del hecho veda un tipo de Estado basado en iguales o similares presupuestos, independientemente del lugar geográfico donde se produjera. A partir del Holocausto, el Estado de Derecho debe ser repensado. El Holocausto no sólo forma parte de la memoria colectiva alemana, sino que es una pieza fundamental de la memoria colectiva europea. La memoria co-

51 J. Habermas, Eine Art Schadensabwicklung, Suhrkamp, 1987.

52 GOLDHAGEN se separa de las tesis expuestas por H. ARENDT del miedo colectivo y sostiene que buena parte del pueblo alemán fue un verdugo voluntario de las acciones de Hitler. No existió miedo sino voluntad. GOLDHAGEN abunda en ejemplos; batallones voluntarios, entrenamientos demasiados breves para socializar a gente adulta, etc. Sin ser un historiador profesional y por ello no tener más elementos de juicio que la lectura atenta del libro, creo que abundan los rumores y que se basa en exceso en los testimonios personales. Ahora bien, en este libro, valiente y decisivo para una época, hay un fondo de verdad insoslayable. D. GOLDHAGEN, Los verdugos voluntarios de Hitler, Taurus, 1997. 
lectiva no se limita a los estrechos márgenes del Estado-nación; puede ser predicada de cualquier grupo social ${ }^{53}$, podría — y debe - ser atribuida al espacio europeo, a la cosmópolis. Existe un tipo de Estado que no es moralmente repetible; existe un tipo de «pegamento social» que no se debe volver a emplear jamás, ya sea en el Estado-nación o en las estructuras posnacionales: éste es el étnico, el racial.

Llama la atención que HABERMAS se mantenga —en algunas ocasiones- tan escéptico respecto a la Constitución europea, pues resulta palmario que podría dibujar un marco adecuado para el patriotismo constitucional de cuño europeo. Como ya se ha puesto de manifiesto, parece tener más confianza en la «opinión pública» que en el Tratado-Constitución europea. Quizá porque, a pesar de la euforia que en algunos ha despertado este texto, HABERMAS incide en la necesidad de que se produzca «un proceso inclusivo de comunicación informal de masas» ${ }^{54}$. La relación entre democracia/ Estado no es exclusiva del Estado-nación. Puede ser extensible a Europa; para ello se deben producir una serie de circunstancias: la emergencia de una sociedad civil europea, la construcción de una esfera pública europea y la formación de una cultura política que pueda ser compartida por todos los ciudadanos europeos.

Parece, pues, que el patriotismo constitucional habermasiano requiere de la construcción previa de una esfera pública que, aunque más difusa que la del Estado-nación, garantice la igual participación de todos los ciudadanos en la construcción europea: es la conclusión inmediata que parece desprenderse de la «comunidad ideal de diálogo» trasplantada a lo normativo. HABERMAS ha dedicado todo un libro a esta transposición: Facticidad y Validez ${ }^{55}$. A él habrá que acudir. Sea suficiente, por ahora, lo expuesto. Desde luego lleva razón BAUMAN al aseverar que «nadie se sentirá sobrecogido por el "patriotismo constitucional"», el cual se distancia de «una versión emergente y desintoxicada de los sentimientos nacionales y colectivos» ${ }^{56}$.

\subsection{Patriotismo cívico de N. MACCORMICK}

El nacionalismo cívico de MACCORMICK posee un marco de referencia distinto al de la Unión Europea; trata de establecer una relación, lo menos conflictiva posible, entre el Reino Unido y Escocia. Un nacionalismo moderado que articule estas relaciones es la meta de MACCORMiCK. Como se ha apuntado, en su libro Quostinig Soverygnty, se hace alusión al papel que la Unión Europea juega en la disolución del concepto clásico de soberanía, y cómo, tal vez, éste sea un buen momento para reivindicar otro tipo de soberanía ${ }^{57}$.

53 «...podemos hablar de memoria colectiva cuando evocamos un hecho que ocupaba un lugar en la vida de nuestro grupo y que hemos planteado o planteamos ahora en el momento en que lo recordamos, desde el punto de vista de ese grupo», M. HALBWACHS, La memoria colectiva, en I. SANCHO ARROYO (trad.), Prensa Universitaria de Zaragoza, 2004, p. 36.

54 J. HABERMAS, «¿Por qué...?», cit., p. 16.

55 Especialmente interesante es la construcción de la comunidad ideal de diálogo en el capítulo III de Facticidad y Validez.

56 Z. BAUMAn, Europa. Una aventura inacabada, cit., p. 42.

57 «According to the argument of this book so far, the European Union has brought about a new form of legal and political order in Western Europe. It has created the possibility of politics "beyond the sovereign state"». 
La expresión «European Commonwealth», contenida en el subtítulo de esta obra libro, parece dejar las cosas claras: ¿Hasta qué punto es posible repetir el modelo de relación que existe entre el Reino Unido y los países pertenecientes a la Commonwealth para Europa? Éste fue un sistema nacido de la colonización y de la conquista que, en principio, no presenta muchos paralelismos con el nacimiento de la Unión Europea. Tampoco parece ser ésta la tesis de MACCORMICK: él defiende desde luego una confederación de carácter voluntario y sui generis. Aunque, a veces, se realicen afirmaciones un tanto extrañas en relación con los imperios ${ }^{58,59}$.

De todos modos, MACCORMicK defiende una nueva concepción de la soberanía, ganando el barlovento al nacionalismo esencialista, pero, a la par, uniendo al ciudadano con los otros y con un territorio concreto; el nacionalismo cívico:

«Under a civil conception of the nation, the nation which exercises the right of self-determination is constituted by a sense of common belonging among those who share civil institutions, with no exclusiveness towards any person or group willing to participate in them» ${ }^{60}$.

El nacionalismo cívico se opone a un nacionalismo de carácter étnico; este último concibe a la nación o al pueblo como portadora de un patrimonio cultural distinto o incluso, en algunos casos, se reivindica un patrimonio genético propio. MACCORMICK apuesta, sin dudas, por el primero: un nacionalismo moderado, que presenta algunas concomitancias con la idea del patriotismo constitucional habermasiano; pero también algunas diferencias.

1. El patriotismo constitucional es una construcción que se mueve en un plano eminentemente normativo o, mejor dicho, parte de una separación entre el Sein y el Sollen, a pesar de entenderlo como un elemento emotivo, como ha hecho últimamente HABERMAS, rezuma influjo kantiano ${ }^{61}$. En el aspecto empírico se respalda en la opinión pública; mas la esfera de lo fáctico no puede ser por sí sola un elemento constitutivo de la nación o del pueblo. Las naciones, los pueblos, al ser ficciones jurídicas, sólo existen en el plano normativo ( «Andererseits entstehen Völker mit ibrem staatlichen Verfassung» ${ }^{62}$ ). Se puede decir más alto; pero no más claro. Sin embargo, MACCORMicK, sin llegar a mezclar los dos planos e influido posiblemente por su institucionalismo, más cercano, a nuestro juicio, a un enfoque sociológico del Derecho, afirma que los ordenamientos normativos pueden tener una «objective existence, not unconnected to states of momentary consciousness of individuals, but not simple constituted by these» ${ }^{63}$.

N. MACCORMICK, Questioning Sovereignty. Law, State, and Nation in the European Commonwealth, Oxford University Press, 2001, p. 167.

58 «There is a difficulty to be faced: multi-nacionality and mult-ethnicity have always been features of empires, and empires at their best have treated ethnicity plurality in a reasonable and tolerant way, though usually with a very least a tacit (sic!) assumption of the superiority of the metropolitan».

59 Para concluir con esta nota sobre la idea de «Imperio»/«Commowealth» quisieramos señalar que nos parece más interesante el intento de BECK y GRANDE de tratar de salir de la idea de imperio como dominio, tal y como la definió JELLINEK y WEBER. Estos autores optan por presentar una idea de «imperio» contra-fáctica, en la que predominan la idea de consenso frente a la de hegemonía, ausencia de jerarquía y que busca la participación de todos sus miembros. U. BECK y E. GRANDE, Das kosmopolitische Europa, Suhrkamp, 2004, p. 90.

60 N. MacCormick, Questioning Sovereygnty, p. 170. Las cursivas son mías.

${ }^{61}$ J. Habermas, Zwischen Naturalismus und Religión, Suhrkamp, 2006, p. 109.

62 J. HABERMAS, Zeit der Übergänge, Suhrkamp, 2001, p. 101.

63 N. MACCORMICK, Questioning Sovereignty, p. 173. 
2. Aquí nos parece se cuela un gazapo «of momentary consciousness of individuals» y —creemos—, para ser coherente con su discurso, debería haber añadido «or groups» — claro que los grupos, que sepamos, no tienen consciencia-. Existe una importante diferencia entre el patriotismo constitucional y el nacionalismo cívico. HABERMAS - compruébense las críticas que aduce en el prólogo de la edición alemana de la obra de C. TAYLOR «Die Annerkenung des Anderen» a este autor- es en lo normativo y, más concretamente, en relación con los derechos fundamentales, un individualista ${ }^{64}$. MACCORMICK incluye, como se aprecia en la definición de nacionalismo cívico citada más arriba, tanto a los grupos como a los individuos que «quieran participar en ella [en la nación]». El tenor del art. I-1 y el principio de subsidiariedad parecen otorgarle la razón. Creemos, sin embargo, que los argumentos de HABERMAS son más sólidos. Además no parece que la Unión Europea preste mucha atención a otros sujetos colectivos que los Estados-nación; buena prueba de ello es que la representación que algunos Ländern o regiones pueden tener en el Consejo es en calidad de representación de todo el Estado-nación al que pertenecen: nimio consuelo ${ }^{65}$. Asimismo el «Comité de las regiones» es meramente consultivo.

3. Por último el nacionalismo cívico, al menos en principio, no se debe oponer al étnico. Esto nos parece un tanto preocupante. A juicio de MACCORMICK, estas construcciones, en el ámbito teórico, se pueden diferenciar, pero, de hecho, en la realidad, hay elementos de uno y de otro en las construcciones nacionalistas: lo que acabamos de arrojar por la ventana entra por la puerta principal, ¿o no? El límite para que esto no suceda es dar prioridad, como hace el autor escocés, a los derechos individuales frente a los colectivos, ubicando a aquéllos como límite frente al uso de éstos.

Patriotismo constitucional y nacionalismo cívico presentan, pues, más concomitancias que diferencias y, a nuestro juicio, pueden constituir caminos interesantes para explorar, para construir un vínculo razonable tanto entre los ciudadanos e instituciones europeas.

Toda palabra posee una deriva que la ha ido trasformando. En el caso de los «conceptos jurídicos» esta afirmación gana aún más predicamento. Por ello, las cuestiones nominales no suelen ser en absoluto indiferentes en el campo del Derecho. En el caso del término «Constitución europea» éste parece evocar a un «territorio», a un concepto de «ciudadanía» y a una «distribución de competencias» más decimonónicas que actuales. Esta misma constelación de significados es la que manejan los que defienden su carácter de Tratado. Creo que es necesario huir tanto de una postura como de otra; hay que percibir que el contenido que otorga sentido a la expresión «Constitución europea» no debe ser el mismo que tradicionalmente se le ha concedido a este término en el Estado-nación. No hay conceptos absolutos ni a-históricos. La esfera pública europea, el nexo entre ciudadanía y territorio plantea nuevos retos — por ejemplo, el contenido en la tesis del no-demos- que requieren soluciones relativamente novedosas e imaginativas, las cuales han de ser buscadas en el marco de las nuevas realidades

${ }^{64}$ J. Habermas, en C. TAYlor, Multikulturalismus und die Politik der Anerkennung, Frankfurt, Fischer, 1993.

${ }_{65}$ «En el Consejo de Ministros de la Unión no cabe la representación de una región o de varias, sino que el representante, aún pudiendo ser regional, representa al Estado en su conjunto». A. MANGAS MARTín, La Constitución europea, cit., p. 110. 
posnacionales y no por contraste con las arbitradas para el Estado-nación -lo que no significa que algunas de estas soluciones presenten una relación de continuidad con el viejo y tradicional Estado-nación—; si no tenemos presente este extremo, estaremos, a nuestro juicio, luchando contra molinos de viento con armas conceptuales ciertamente obsoletas, tendremos la derrota asegurada y, lo que se nos antoja aun más grave, perderemos el sentido de la realidad. 